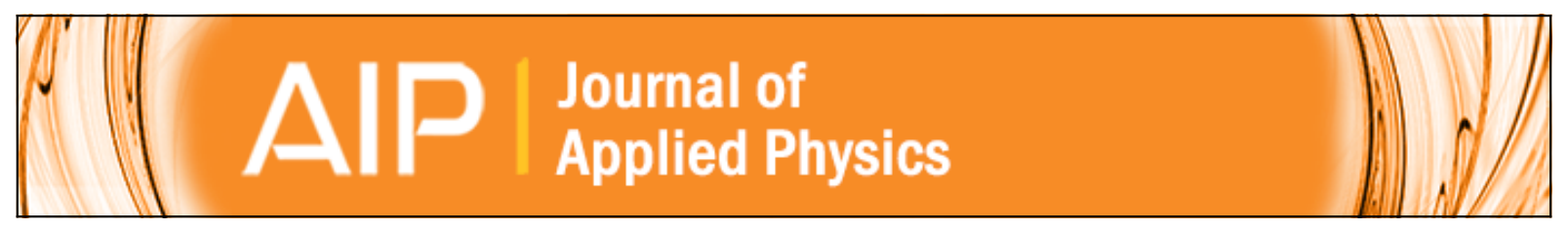

\title{
Modification of single-walled carbon nanotube electrodes by layer-by-layer assembly for electrochromic devices
}

Vaibhav Jain, Henry M. Yochum, Reza Montazami, James R. Heflin, Liangbing Hu, and George Gruner

Citation: Journal of Applied Physics 103, 074504 (2008); doi: 10.1063/1.2891256

View online: http://dx.doi.org/10.1063/1.2891256

View Table of Contents: http://scitation.aip.org/content/aip/journal/jap/103/7?ver=pdfcov

Published by the AIP Publishing

\section{Articles you may be interested in}

Enhancement of efficiency of a conducting polymer P3HT:CdSe/ZnS quantum dots hybrid solar cell by adding single walled carbon nanotube for transporting photogenerated electrons

J. Renewable Sustainable Energy 5, 033107 (2013); 10.1063/1.4807475

Study on the performance of carbon nanotube-based electrochromic cell AIP Conf. Proc. 1502, 381 (2012); 10.1063/1.4769158

Electrical characterization of single-walled carbon nanotubes in organic solar cells by Kelvin probe force microscopy

Appl. Phys. Lett. 96, 083302 (2010); 10.1063/1.3332489

Millisecond switching in solid state electrochromic polymer devices fabricated from ionic self-assembled multilayers

Appl. Phys. Lett. 92, 033304 (2008); 10.1063/1.2834818

An electrochromic film device to teach polymer electrochemical physics

Am. J. Phys. 75, 839 (2007); 10.1119/1.2746365

\section{MIT LINCOLN} LABORATORY CAREERS

Discover the satisfaction of innovation and service to the nation
- Space Control

- Air \& Missile Defense

- Communications Systems \& Cyber Security

- Intelligence, Surveillance and

Reconnaissance Systems

$$
\begin{aligned}
& \text { - Advanced } \\
& \text { Electronics } \\
& \text { - Tactical Systems } \\
& \text { - Homeland } \\
& \text { Protection } \\
& \text { - Air Traffic Control }
\end{aligned}
$$

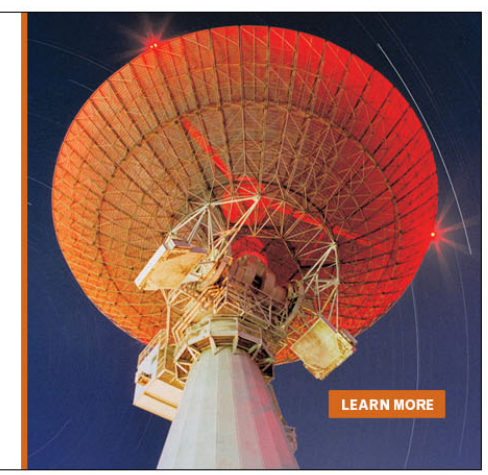




\title{
Modification of single-walled carbon nanotube electrodes by layer-by-layer assembly for electrochromic devices
}

\author{
Vaibhav Jain, ${ }^{1, a)}$ Henry M. Yochum, ${ }^{2}$ Reza Montazami, ${ }^{3}$ James R. Heflin, ${ }^{3}$ \\ Liangbing $\mathrm{Hu},{ }^{4, a), b)}$ and George Gruner ${ }^{4}$ \\ ${ }_{1}^{1}$ Macromolecular Science and Engineering, Virginia Tech, Blacksburg, Virginia 24061, USA \\ ${ }^{2}$ Department of Physics and Engineering, Sweet Briar College, Sweet Briar, Virginia 24595, USA \\ ${ }^{3}$ Department of Physics, Virginia Tech, Blacksburg, Virginia 24061, USA \\ ${ }^{4}$ Department of Physics and Astronomy, University of California, Los Angeles, California 90095, USA
}

(Received 10 October 2007; accepted 12 January 2008; published online 3 April 2008)

\begin{abstract}
We have studied the morphological properties and electrochromic (EC) performance of polythiophene multilayer films on single wall carbon nanotube (SWCNT) conductive electrodes. The morphology for different numbers of layer-by-layer $(\mathrm{LbL})$ bilayer on the SWCNT electrode has been characterized with atomic force microscopy and scanning electron microscope, and it was found that the LbL multilayers significantly decrease the surface roughness of the nanoporous nanotube films. The controlled surface roughness of transparent nanotube electrodes could be beneficial for their device applications. We have also fabricated EC devices with LbL films of poly[2-(3-thienyl) ethoxy-4-butylsulfonate/poly(allylamine hydrochloride) on SWCNT electrodes, which not only have high EC contrast but also sustain higher applied voltage without showing any degradation for more than 20000 cycles, which is not possible in the case of indium tin oxide electrodes. Cyclic voltammetry of the LbL films formed on SWCNT shows higher current at low potential, revealing the feasibility of SWCNT electrode as a good host for electrolyte ion insertion. (C) 2008 American Institute of Physics. [DOI: 10.1063/1.2891256]
\end{abstract}

\section{INTRODUCTION}

Thin single wall carbon nanotube (SWCNT) networks with thickness in the range of $10-100 \mathrm{~nm}$ have high sheet conductance while maintaining high optical transparency. ${ }^{1-3}$ These transparent electrodes have been used in organic solar cells, photovoltaic devices, and light emitting diodes, which show comparable device performance with indium tin oxide (ITO), along with better mechanical properties. ${ }^{4-6}$ SWCNT films are porous and have high surface area, which can be either bane or boon for the device application. The larger surface area ensures the availability of a higher amount of reactive sites at the SWCNT surface, but due to the rough nature of the film, charge injection and light intensity can both be nonuniform and can occasionally shorten the device lifetime. ${ }^{7}$ To overcome this problem, we adopted the approach of coating the SWCNT film with the layer-by-layer (LbL) assembly; this helps in reducing the surface roughness and integrating the SWCNT into thin-film optoelectronic devices without short-circuiting the electrodes.

To achieve this, conformable coating of another layer is critical. Other techniques such as spin coating, solution casting, or electropolymerization, where rapid deposition of the material occurs, do not result in smooth films on rough surfaces. The Langmuir-Blodgett technique is one possible choice for developing such morphology, but it is preferred for flatter surfaces; the thickness of its individual layers cannot be varied as it is determined by the length of the molecule, it cannot smooth the surface, and also the electroac-

\footnotetext{
${ }^{a)}$ These two authors contributed equally to this work.

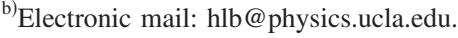

tivity decreases for thicker films. ${ }^{8}$ In contrast to this, LbL deposited films not only decrease the surface roughness for uniform charge injection but also forms a nanoporous morphology which offers low resistance to charge and mass transfer.

Electrochromic (EC) devices undergo reversible color change in a material by the application of external voltage. ${ }^{9}$ LbL is also an excellent processing tool in the development of EC devices, as it provides flexibility and precise control over designing EC films on a diverse array of substrates with high uniformity and thickness control, higher contrast by the combination of multiple EC materials, and an increase in ionic conductivity for faster switching speeds. ${ }^{10} \mathrm{LbL}$ films coated on SWCNT electrodes could potentially lead to better overall EC performance as compared to LbL films on ITO electrodes because of enhanced ion insertion, voltage sustainability, and increased life cycles. Several different groups have deposited modified carbon nanotubes on various substrates for different applications by the ionic LbL approach. ${ }^{11-14}$ In the present work, we have used LbL assembly on separately deposited conducting, transparent SWCNT electrodes rather than incorporate SWCNTs into LbL films. Carrillo et al. ${ }^{15}$ have used the LbL technique to coat the SWCNT surface with gold nanoparticles and polyelectrolytes, but their approach is more tedious and requires several steps of cross-linking. In addition, they deposited SWCNT through the chemical vapor deposition process, requiring catalysts for nanotube growth, which results in a nonuniform, low conductivity surface; hence, LbL deposition is random and unsuitable for electronic applications. In contrast, we have used a very well established technique of depositing uniform, highly conductive SWCNT films on large areas 

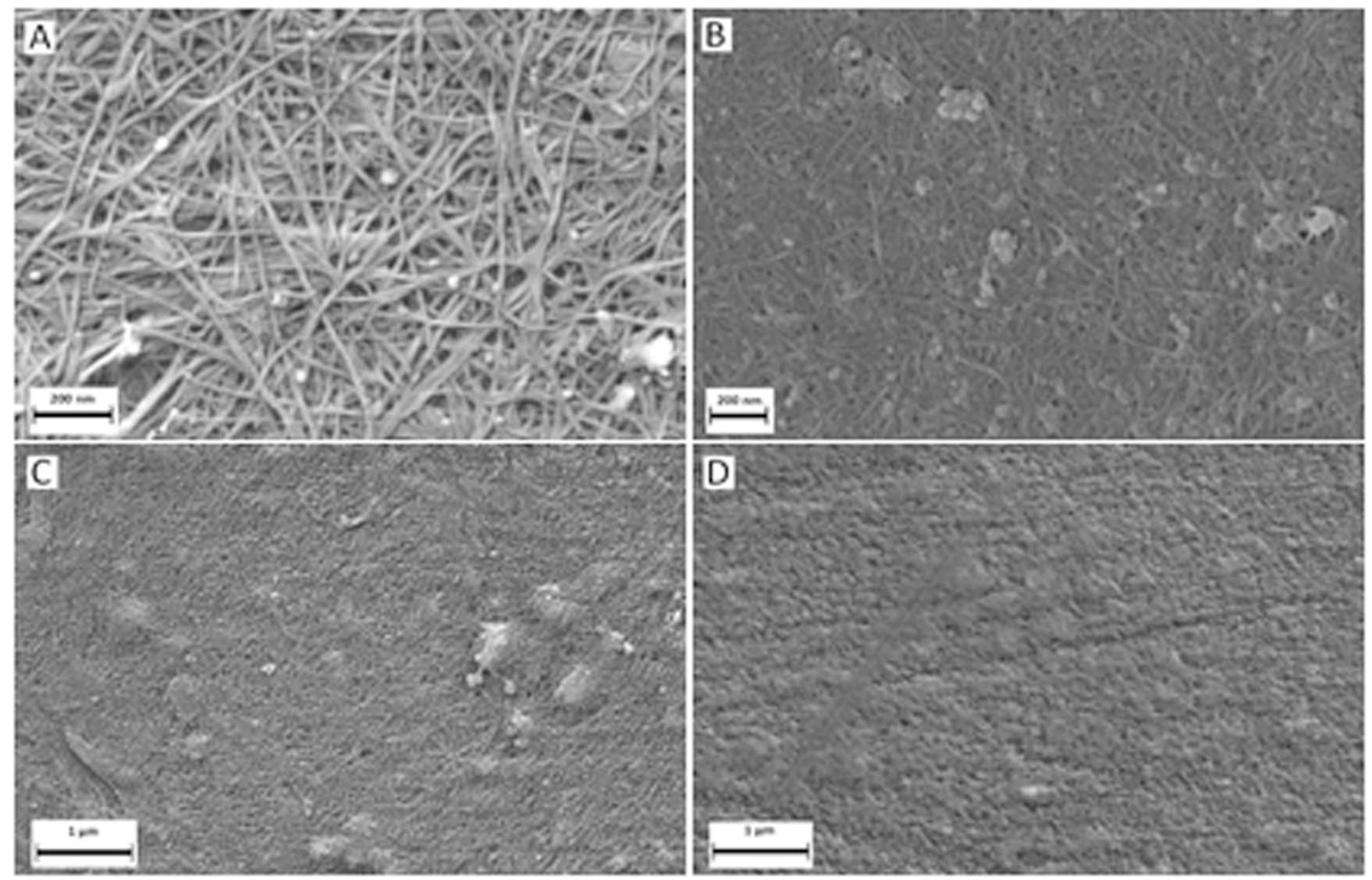

FIG. 1. (Color online) Scanning electron microscopy images of CNT electrode with (a) no film, (b) two bilayers, (c) five bilayers, and (d) ten bilayers of $\mathrm{PAH} / \mathrm{PTEBS}$ film.

through spray coating ${ }^{16}$ and combined the benefits of that method with a bottom-up LbL approach for highly stable EC devices.

\section{EXPERIMENT}

SWCNT films on the glass have been deposited by spray coating with a similar procedure as explained in one of our previous work. ${ }^{16}$ Briefly arc-discharged single wall nanotube (Carbon Solutions Inc.) are dispersed in water with $0.5 \%$ sodium dodecyl sulfate with surfactants of $1 \mathrm{mg} / \mathrm{ml}$. The solutions were sprayed onto glass or plasticsubstrates which were then heated to $80{ }^{\circ} \mathrm{C}$. The water-soluble sodium polythiophene poly[2-(3-thienyl) ethoxy-4-butylsulfonate] (PTEBS) (Ref. 17) (American Dye Source) was used as the active EC material and bilayers were fabricated by the LbL deposition technique. EC PTEBS LbL devices have been characterized recently and good green to orange-red color changing properties have been obtained. ${ }^{18}$ Glass slides with a thin film of single-walled nanotubes were used as the substrate in the present work. The LbL deposition was performed by alternately dipping the SWCNT electrode in a positively charged aqueous polymeric solution, poly(allylamine hydrochloride) (PAH) (Sigma-Aldrich, $M_{w} \sim 75000$, $10 \mathrm{mM}$ concentration, $p \mathrm{H} \mathrm{4}$ ), and negatively charged polymeric solution, PTEBS ( $1 \mathrm{mM}, p \mathrm{H} \mathrm{4})$, for $6 \mathrm{~min}$ and rinsing in de-ionized water for $2 \mathrm{~min}$.

\section{RESULTS AND DISCUSSION}

Morphological studies of different numbers of PAH/ PTEBS bilayers on SWCNT electrode by field emission scanning electron microscope (FESEM-LEO 1550) (Fig. 1) at $5 \mathrm{kV}$ and atomic force microscope (AFM) (Nanoscope IVa) (Fig. 2) reveals that the LbL films are conformably coated onto the surface of the nanotube substrate. The AFM imaging was done in tapping mode with a cantilever of $50 \mathrm{~N} / \mathrm{m}$ force constant and image size of $2 \times 2 \mu \mathrm{m}^{2}$ and $0-60 \mathrm{~nm} z$ scale. Due to the three-dimensional nanomesh network topology, the bare SWCNT electrodes with varied thickness of nanocomposite multilayers with pores show intriguing morphological properties. AFM scans of several LBL films confirmed that the films start wrapping around the nanotubes for the first few bilayers (two) and become a thick cylindrical film sheet around the nanotubes for a slightly higher number of bilayers (five); repeated scans were done at different locations to ensure that the morphological properties are similar throughout the sample. As can be clearly seen, the structure of the surface changes after deposition of a few bilayers and the film surface morphology smoothens as compared to the bare SWCNT electrode. As material is deposited in each bilayer, moving from five to a higher number of bilayers (eight), the thin cylindrical sheets joined together to yield a nanoporous morphology. Sometimes, but not always, a few holes in the LbL film with more than eight bilayers were found. Continuation of the deposition to ten 

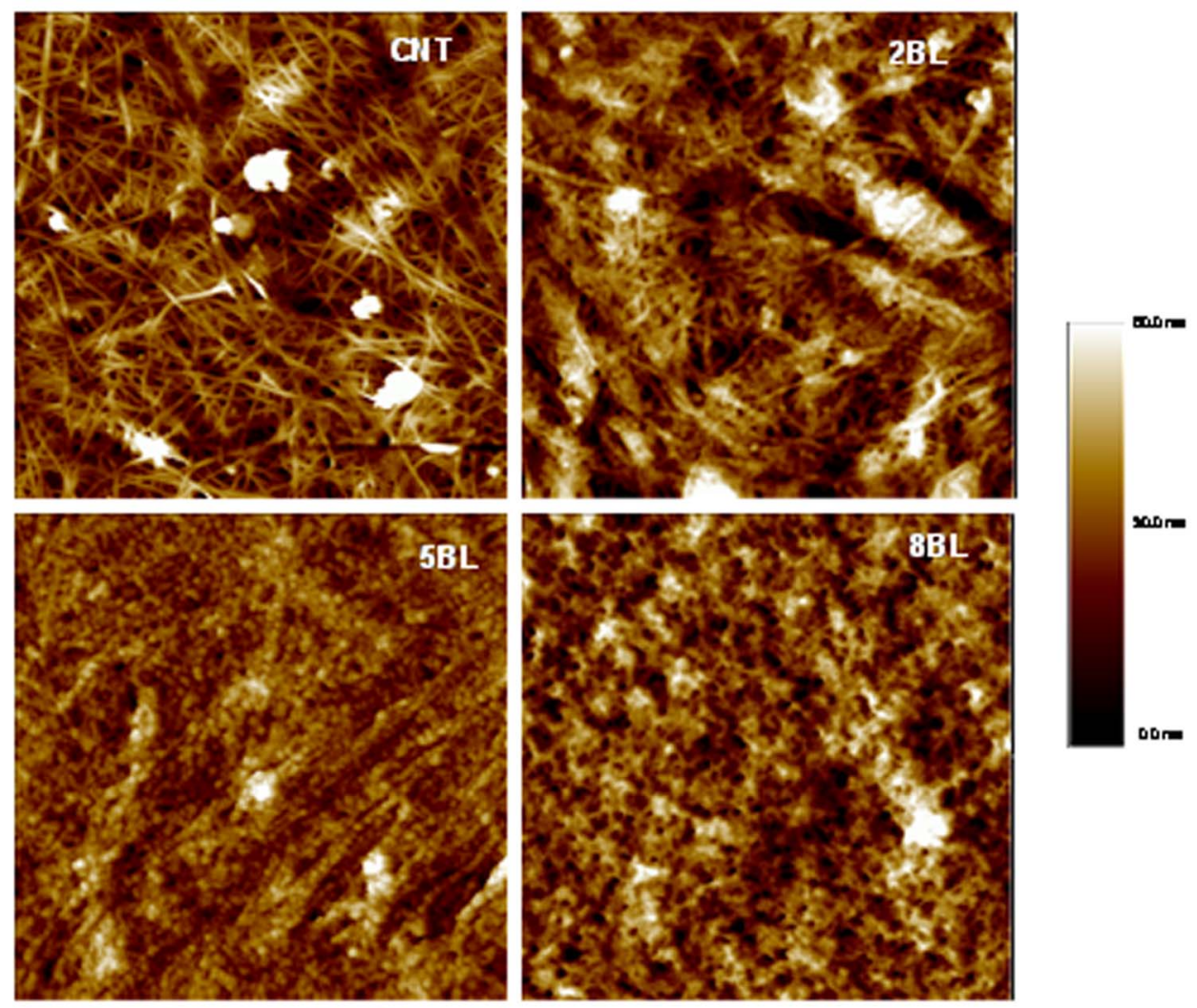

FIG. 2. (Color online) AFM height images of bare CNT electrode and film with two, five, eight, and ten bilayers. Area is $2 \times 2 \mu \mathrm{m}^{2}$ and the $z$ scale is from 0 to $60 \mathrm{~nm}$. Also represented is the average surface roughness plot for different numbers of bilayers.
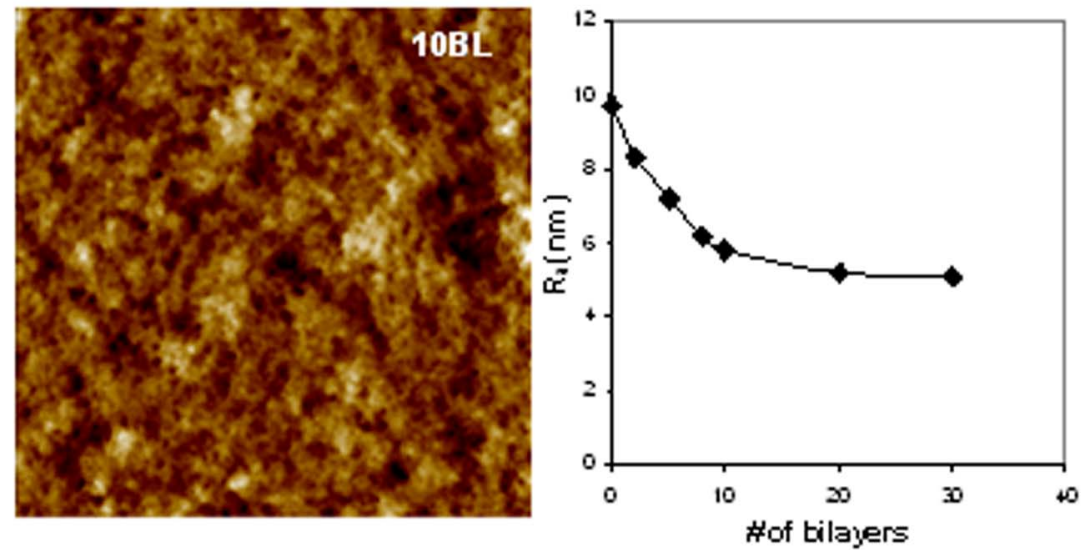

bilayers keeps the porosity in the film architecture but also increases the smoothness in the film. The plot of the surface roughness versus number of bilayers shows that the overall average surface roughness $\left[R_{a}(\mathrm{~nm})\right]$ of the deposited film decreases as the film thickness increases for higher numbers of bilayers; the bare SWCNT electrode has the highest surface roughness, which becomes nearly constant for films with ten or more bilayers. The decrease in surface roughness is caused by the subsequent multilayer deposition, which fills the voids in the SWCNT electrode and makes the surface smoother

To study the redox activity of PAH/PTEBS multilayers on the SWCNT film, cyclic voltammetry (CV) experiments were performed with a computer-controlled potenstiostat/ galvanostat (PINE AFCBP1 bipotentiostat); all measurements were carried out in $\mathrm{LiClO}_{4}(0.1 M)$ /acetonitrile with platinum $(\mathrm{Pt})$ and $\mathrm{AgCl} / \mathrm{Ag}$ as counterelectrode and reference electrode, respectively. The bare CNT electrode shows no electroactivity (Fig. 3(a)) while the PAH/PTEBS LbL films clearly have a redox peak at around $+0.10 \mathrm{~V}$. The oxidation peak for the spin coated PTEBS film at the same conditions is at $+0.6 \mathrm{~V}$, but here the peak has shifted to a lower potential which reduces the impedance of the EC film and increases the electron transport efficiency. ${ }^{14}$ This is attributed to the highly porous nature of the SWCNT film. The peak did not skew to the lower peak voltage on increasing the scan rate, which suggests the negligible internal resistance of the LBL films and faster diffusion processes. The diffusion-controlled redox process was confirmed by a linear increase of current density peak with the square root of scan rate (Fig. 3(b)) for a 40-bilayer film of PAH/PTEBS. It is important to note that the diffusion-controlled process provides maximum access to the film, ${ }^{19}$ which is attributed to complete ion intercalation and facile movement of ions in and out of the system and also brings long term stability (>20 000 cycles) to the device. The problems of ion porosity and diffusion have been observed with ITO electrodes as the low surface area brings more resistance for ionic move- 

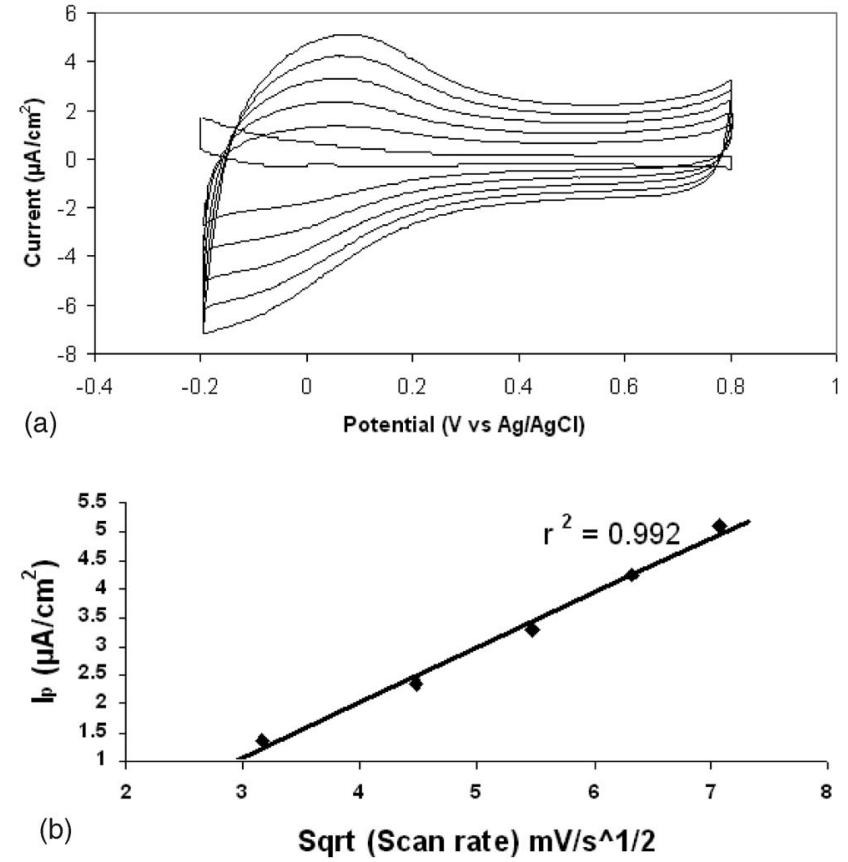

FIG. 3. (a) $\mathrm{CV}$ of the bare $\mathrm{CNT}$ electrode at $10 \mathrm{mV} / \mathrm{s}$ and 40-bilayer film of $\mathrm{PAH} / \mathrm{PTEBS}$ at $5,10,15$, and $20 \mathrm{mV} / \mathrm{s}$. (b) The linear relationship of peak current with the square root of scan rate.

ment and in some cases, the electrochemical reaction moves toward a surface controlled process rather than diffusion controlled. $^{20}$

The higher surface charge concentration of EC material on the SWCNT electrode is also confirmed by the large amount of charge intercalated in the system. The overall charge density $(Q)$ calculated from CV experiment for PAH/ PTEBS LbL film is $55 \mu \mathrm{C} / \mathrm{cm}^{2}$ for each bilayer deposited. The high PTEBS (PAH is electrochemically inactive) surface coverage for each bilayer calculated by $\Gamma=Q / n F(F=96.5$ $\times 10^{3} \mathrm{C} / \mathrm{mol}$ and $n=1$ is the number of electrons transferred) is $5.73 \times 10^{-9} \mathrm{~mol} / \mathrm{cm}^{2}$, which is much higher $\left(\sim 10^{-10}-10^{-11} \mathrm{~mol} / \mathrm{cm}^{2}\right)$ than the average surface coverage of polyelectrolytes for other LBL systems ${ }^{21}$ on ITO electrodes.

The spectroelectrochemistry performance of EC devices on SWCNT electrodes has been evaluated by Perkin-Elmer Lambda 25 UV-visible spectrophotometer. Absorption spectra at different applied potentials $(0.75-2.0 \mathrm{~V})$ were measured with the EC LbL film on SWCNT electrode placed in a clear glass cuvette in $0.1 \mathrm{M} \mathrm{NaClO}$ (aq) electrolyte solution. The base line was taken using a bare SWCNT electrode with no LbL film on it, which in itself has more than $80 \%$ transmission as compared to glass over a broad spectral range from visible to infrared. The in situ transmission spectrum (Fig. 4) of a 40-bilayer PAH/PTEBS film on SWCNT film shows high EC contrast of 50\% between the orange-red (bleached) state at $0 \mathrm{~V}$ and the dark green (colored) state at $2 \mathrm{~V}$ at $\lambda_{\max }$ of $735 \mathrm{~nm}$. The EC device has no optical memory, and hence the film loses color as soon as the voltage supply is turned off. On stepwise increase in potential from 0.75 to $2.0 \mathrm{~V}$, the absorption decreased at $410 \mathrm{~nm}$ due to the $\pi-\pi^{*}$ transition of PTEBS and increased at the wave-

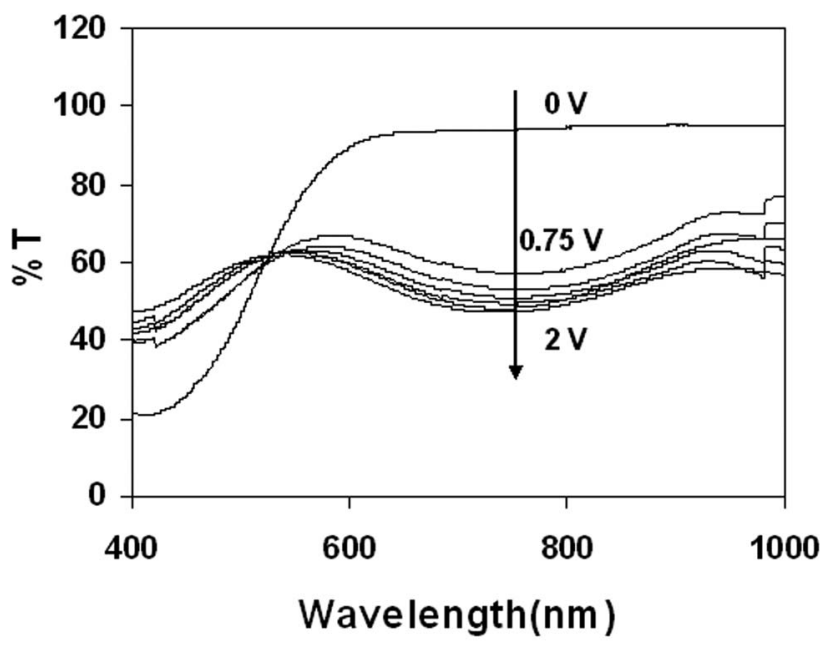

FIG. 4. Change in transmission spectra of 40-bilayer PAH/PTEBS film on application of $0 \mathrm{~V}$ and step increase in voltage from 0.75 to $2.0 \mathrm{~V}$.

length of $735 \mathrm{~nm}$ due to polaronic transitions. The contrast did not increase further for the application of $>2.0 \mathrm{~V}$, but the device was completely reversible for as high as $8-10 \mathrm{~V}$ of applied potential. This is in contrast to EC films on ITO, which suffer from the problem of not sustaining high voltages, leading to burning or degradation of the EC film. The EC coloration efficiency ${ }^{7}[\Delta \mathrm{OD} / \Delta Q$, where the optical density $\Delta \mathrm{OD}$ is $\log \left(T_{\text {bleach }} / T_{\text {colored }}\right)$ at a specific wavelength] calculated for the EC device (40 bilayers) was found to be $141 \mathrm{~cm}^{2} \mathrm{C}^{-1}$ at $735 \mathrm{~nm}$ and is comparable to that obtained in related polythiophene materials. ${ }^{22}$

\section{CONCLUSION}

The LbL assembly on SWCNT film has been shown to effectively reduce the surface roughness as well as decrease the impedance and, based on CV measurements, increase the efficiency of ion transport. The improvement of the surface roughness by LbL modification may potentially improve the device performance of organic photovoltaic and organic light emitting diode devices with transparent SWCNT electrodes as anodes by improving the uniformity of charge injection. The high contrast $(\sim 50 \%)$ in our EC devices for long switching cycles and the ability to operate work at higher potential combined with a flexible substrate ${ }^{18}$ make it a good candidate for next generation e-paper displays as compared to flexible ITO substrates. The results show that the high surface coverage and charge of polyelectrolyte multilayers make the LbL-modified SWCNT film more tailored to specific application along with lower costs and environmentally friendly processing conditions. Future work will include a detailed study of EC and supercapacitor devices using such LBL-modified transparent SWCNT electrode.

\section{ACKNOWLEDGMENTS}

The authors are very grateful to Dr. Anil Kumar and Rabindra Sahoo of the Department of Chemistry at the Indian Institute of Technology (IIT), Mumbai, for providing the CV data. H. M. Yochum acknowledges support from the Sweet Briar College Faculty Fellowship. 
${ }^{1}$ Y. Zhou, L. Hu, and G. Grüner, Appl. Phys. Lett. 88, 123109 (2006). ${ }^{2}$ Z. Wu, Z. Chen, X. Du, J. M. Logan, J. Sippel, M. Nikolou, K. Kamaras, J. R. Reynolds, D. B. Tanner, A. F. Hebard, and A. G. Rinzler, Science 305, 1273 (2004).

${ }^{3}$ D. S. Hecht, L. Hu, and G. Grüner, Appl. Phys. Lett. 89, 133112 (2006).

${ }^{4}$ M. W. Rowell, M. A. Topinka, M. D. McGehee, H. Prall, G. Dennler, N. S. Sariciftci, L. Hu, G. Grüner, Appl. Phys. Lett. 88, 233506 (2006).

${ }^{5}$ D. Zhang, K. Ryu, X. Liu, E. Polikarpov, J. Ly, M. E. Tompson, and C. Zhou, Nano Lett. 6, 1880 (2006).

${ }^{6} \mathrm{~J} . \mathrm{Li}, \mathrm{L} . \mathrm{Hu}, \mathrm{L}$. Wang, Y. Zhou, G. Grüner, and T. J. Marks, Nano Lett. 6, 2472 (2006).

${ }^{7}$ R. A. Hatton, N. P. Blanchard, A. J. Miller, and S. R. P. Silva, Physica E (Amsterdam) 37, 124 (2007).

${ }^{8}$ G. Decher, Science 227, 1232 (1997).

${ }^{9}$ P. M. S. Monk, R. J. Mortimer, and D. R. Rosseinsky, Electrochromism: Fundamentals and Applications (VCH, Weinheim, 1995).

${ }^{10}$ G. Decher and J. D. Hong, Makromol. Chem., Macromol. Symp. 95, 321 (1991).

${ }^{11}$ H. Paloniemi, M. Lukkarinen, T. Aaarito, S. Areva, J. Leiro, M. Heinonen, K. Haapaka, and J. Lukkari, Langmuir 22, 74 (2006).
${ }^{12}$ K. J. Loh, J. Kim, J. P. Lynch, N. Wong, S. Kam, and N. A. Kotov, Smart Mater. Struct. 16, 429 (2007).

${ }^{13}$ J. H. Rouse and P. T. Lillehei, Nano Lett. 3, 59 (2003).

${ }^{14}$ M. Zhang, L. Su, and L. Mao, Carbon 44, 276 (2006).

${ }^{15}$ A. Carrillo, J. A. Swartz, J. M. Gamba, R. S. Kane, N. Chakarpani, B. Wei, and P. M. Ajayan, Nano Lett. 3, 1437 (2003).

${ }^{16}$ L. Hu, G. Gruner, J. Gong, C. Kim, and B. Hornbostel, Appl. Phys. Lett. 90, 093124 (2007).

${ }^{17}$ Q. Qiao, L. Su, J. Beck, and J. T. Mcleskey, Jr., J. Appl. Phys. 98, 94906 (2005).

${ }^{18}$ V. Jain, H. M. Yochum, R. Montazami, and J. R. Heflin, J. Mater. Chem. (unpublished).

${ }^{19}$ D. DeLongchamp, M. Kastantin, and P. Hammond, Chem. Mater. 15, 1575 (2003).

${ }^{20}$ M. Gratzel, J. Photochem. Photobiol. C 4, 145 (2003).

${ }^{21}$ D. Ingersoll, P. J. Kulesza, and L. R. Faulkner, J. Electrochem. Soc. 141, 140 (1994).

${ }^{22}$ D. M. Welsh, A. Kumar, E. W. Meijer, and J. R. Reynolds, Adv. Mater. (Weinheim, Ger.) 11, 1379 (1999). 